\title{
Practice and Thinking on Graduate Degree Management Information
}

\author{
Hua Zheng ${ }^{1}$ \\ ${ }^{1}$ Graduate School of Jinan University, Guangzhou, Guangdong, 510632, China
}

KEYWORDS: Graduate; Degree Management; Information

\begin{abstract}
Explained by the importance of information management graduate degree, graduate degree analysis of some of the problems of information management practice currently exist in the process, put forward ideas to solve the problem of combining software design theory.
\end{abstract}

\section{Introduction}

With increasing graduate enrollment size and type of growing enrollment, so how will graduate administration liberated from large and repetitive work out, improve management efficiency, postgraduate education and research management will put more energy and strength Training and quality control and other aspects of graduate management became a serious problem. With the development of computer and network technology, many domestic postgraduate education authorities using information technology, management information systems developed using graduate, graduate education of information technology to achieve varying degrees. Degree management as an important part of graduate management, it more needs to implement information management. Establish and improve the graduate degree in information management systems to ensure that timely and accurate information, information sharing, online process management, standardize management degree [2], will greatly improve the efficiency of postgraduate management.

At present, although many colleges and universities have made some achievements in the area of information management graduate degree, but for various reasons degree in management there are still many problems to be solved in terms of information technology, the importance of a graduate degree from the article management information, analyzes the current Graduate design problem management information exists in practice, and the existing problems and propose appropriate solutions to system design ideas.

\section{The Significance of Graduate Degree Management Information}

Graduate degrees awarded are managed by many interrelated elements constitute not only relates to the training process of training programs, accomplishments and other elements by including the degree to apply for grant process; both papers reviewers, but also the respondent records management; both degree applicants It has contained elements of audit management personnel at all levels. Information management of these elements should be within a certain range of time and space to organize scientific and rational, which is to a degree a degree in information management from the application, eligibility review, the paper reviewers result entry, entry and other matters respondent records management extended to information management, quality management, analysis and decision management, assessment management system, etc., all elements of management. 
Graduate degree awarded by the management is composed of many processes. Graduate degree from the application to the final is a degree-granting process, the paper reviewers from reviewer to reviewer arrange entry is a result of the completion of the process, from the respondent to arrange thesis defense site, to generate the respondent recording is a process. Information conducive to the realization of degree-granting management the whole process of management, and the loose ends from the organization to implement the results of the control, the feedback information to form a complete process to form an effective management process.

Degree-granting work management services complex, many links, workload, and various aspects are closely linked, there is no scientific methods and strict rules and regulations cannot guarantee the orderly degree-granting work. Management degree includes a graduate degree in information management, information management and discipline tutor information management, information management is responsible for graduate degree entry degree of information, audit, management, and grant reporting. The basic information to permeate all aspects and degrees awarded throughout, this is a scientific management requirements must be standardized according to certain procedures and this information is conducive to a successful management process. Through the improvement of management information system and management system, standardize management, improve management efficiency and improve the level of standardization and modernization of management degree, the degree-granting management into a virtuous circle.

\section{The Problems in Post-Graduate Degree Management Information Practice}

Management information to improve graduate education management is an important tool, not only the affected system is running system, school culture, rules and regulations and other non-technical factors, but also by the data security, database consistency, data synchronization, concurrent performance technology influencing factors, so a graduate degree must have a management information base information technology, management, basic and applied basic. Graduate students in colleges and universities during the implementation of management information exist mainly in the following design issues:

The design is based on a graduate degree in state and degree-granting process as the main line, or to a graduation process, a degree of two parallel process flow of the main line? Currently used in some colleges and universities graduate degree in management system main line, resulting in a final conclusion with degree graduation reply conclusion intertwined, it is not conducive to extract information and systems analysis degree;

The different aspects of graduate education management of data standards requirements are different, resulting in management systems data standards are not uniform, as well as causing the system data convergence is not on the data information at various stages of the transfer when there will Absence, need to rely on a number of additional data conversion work to make up for the lack of such information;

The degree of information audit approval process is not detailed, only to achieve a result of retroactive, did not establish the appropriate assessment system is not conducive to further analysis and research;

The degree management system decision-making ability is not strong, not only to regulate the management information system information, but also can make full use of information, analysis and study, do not let us in "data rich and information poor" situation;

The management system to serve to stay in the construction of key management personnel, ideas for graduate students and teachers, tutors and services have not been fully realized. 


\section{The Strategies of Graduate Degree Management Information}

Graduate degree in management information for the practice deficiencies, on the one hand by the development, and implementation of the reform of the management system to make up for; through more detailed design of the system and to make up for lack of human hand on a technical level technician from the implementation of the system.

Art should fully understand the school of information technology overall plans and objectives, information technology is to continue to enhance the competitiveness of school, especially to enhance the core competitiveness. From a technical point of view it should be based on the following ideas to design information management system:

Be familiar with degree-granting management process of the process Simplify link, fully consider the degree of information and enrollment information, contact information of the internal culture, planning and overall design to ensure the smooth transfer of the various aspects of the data;

On the system design and implementation to fully consider the management of information literacy, hardware and software expertise to uneven other characteristics, should be targeted to provide a good human-computer interaction, stable operating performance of the system interface;

The information systems for the various degrees involved are using the grant management integration, develop the appropriate system interface, through computer technology and database technology planning, through the establishment of a unified, complete and rational scientific data center, to achieve accurate and timely data synchronization, to ensure the integrity and consistency of the data center, data sharing, degree of information resources unified management, real-time monitoring and reasonable allocation of resources in order to achieve data standardization, systematic management;

The transfer system service focus, focus on clients existing management information systems graduate education management personnel, on the basis of existing features to ensure stable operation, the system to expand the service to shift the focus to the teacher, classroom teachers and graduate students who want to establish a platform for the exchange of instructors, academic exchange platform and student exchange platform for teachers and students to participate in a graduate degree in information management processes to truly reflect the supervisor to graduate education, graduate-centric philosophy;

Increase its research function of the system, on the basis of available information, through further data mining, analysis, provide assistance for decision-making and research management;

The degree in information management, degree status line should be designed as two state lines, a graduation process, a degree process. These two lines have a certain state of mutual restraint, but also independent of each other. According to their type in the graduate degree-granting process, or at the same time to go graduate, degree two processes eventually produce two degrees status: whether the graduate and get a degree; or just take a degree in the process, whether to produce a degree of degree status;

Take full account of existing national codes of professional standards in different phases of the code of professional differences, the establishment of "Enrollment Code," "culture code 'control of relational tables and" degree of code "between the different stages of data conversion between code in order to achieve a degree in information management in the enrollment data, training and integration of data need to be reported in the final degree of data to ensure seamless management of graduate education at different stages of data;

The approval procedures should be refined degree audit, establish appropriate audit approval system for different types of students, collected papers by reviewers scoring system, the respondent rating scale system, evaluation system and other disciplines, finishing detailed data and related 
degree feedback information for the conduct of the quality analysis and evaluation, teaching quality assessment, discipline construction level assessment to provide data support.

\section{Conclusion}

Education information is a process of thinking, concepts of education reform. Management Information is the essence of mining information and knowledge resources to continuously improve work efficiency and management level, to promote reform. In the context of information technology, information is to improve graduate education management level, improve the competitiveness of the basic school, degree management issues open management is an inevitable trend. We must seize the opportunity for a degree in management information, using the Internet, computers and other information technology tools, making management graduate degree real standardization, information technology, efficient, intelligent, realize the transformation Graduate Education.

\section{REFERENCE:}

[1] Li XueJin, The graduate education management information system [J]. Chinese Education Information, 2008, (5): 48-49.

[2] Wu Haiping, Guliang Fei, Zhao Wei. The construction of the model next generation of Graduate Education Information System of [J]. Degrees and Graduate Education, 2006, (12): 27-30.

[3] Pu Fang. Postgraduate Degree Management System provides standardized security [J]. Gansu Science and Technology, 2008, (5): 174-176. 